\title{
Best possible estimates of weak solutions of boundary value problems for quasi-linear elliptic equations in unbounded domains
}

\author{
Damian Wiśniewski
}

\begin{abstract}
We investigate the behaviour of weak solutions of boundary value problems for quasi-linear elliptic divergence second order equations in unbounded domains. We show the boundedness of weak solutions to our problem. Using barrier function and applying the comparison principle, we find the exact exponent of weak solutions decreasing rate near the infinity.
\end{abstract}

\section{Introduction}

Let $B_{1}(\mathcal{O})$ be the unit ball in $\mathbb{R}^{n}, n \geq 3$, with center at the origin $\mathcal{O}$ and $G \subset \mathbb{R}^{n} \backslash B_{1}(\mathcal{O})$ be an unbounded domain with the smooth boundary $\partial G$. We consider the following problem :

$$
\left\{\begin{array}{r}
-\frac{d}{d x_{i}}\left(r^{\tau}|u|^{q}|\nabla u|^{m-2} u_{x_{i}}\right)+a_{0} r^{\tau-m} u|u|^{q+m-2} \\
-\mu_{0} r^{\tau}|u|^{q-1}|\nabla u|^{m} \operatorname{sign} u=f, x \in G ; \\
\alpha(x) r^{\tau}|u|^{q}|\nabla u|^{m-2} \frac{\partial u}{\partial \vec{n}}+\gamma(\omega) r^{\tau-m+1} u|u|^{q+m-2}=g, x \in \partial G ; \\
\lim _{|x| \rightarrow \infty} u(x)=0 ;
\end{array}\right.
$$

Key Words: Elliptic divergence quasi-linear equations, Weak solutions, Unbounded domains, Comparison principle, Barrier function.

2010 Mathematics Subject Classification: Primary 35D30, 35J25, 35J62; Secondary 35A23, 35B40, 35B45, 35B51.

Received: 09.03.2016

Revised: 02.07 .2016

Accepted: 05.07.2016 
BEST POSSIBLE ESTIMATES OF WEAK SOLUTIONS OF BOUNDARY VALUE PROBLEMS FOR QUASI-LINEAR ELLIPTIC EQUATIONS IN UNBOUNDED DOMAINS

here $a_{0} \geq 0,0 \leq \mu_{0}<\frac{q+m-1}{m-1}, q \geq 0,1<m<n, \tau \leq \begin{cases}0, & \text { if } m \geq 2, \\ m-2, & \text { if } m \in(1,2)\end{cases}$ $\gamma(\omega)$ is a positive bounded function and $\alpha(x)=\left\{\begin{array}{ll}0, & \text { if } x \in \mathcal{D} ; \\ 1, & \text { if } x \notin \mathcal{D},\end{array} \quad \mathcal{D} \subseteq \partial G\right.$ is the part of the boundary $\partial G$, where the Dirichlet boundary condition is posed. Thus, if $\mathcal{D}=\partial G$ then we have the Dirichlet problem, if $\mathcal{D}=\emptyset$ then $\alpha(x)=1$ and we have the Robin problem, if $\mathcal{D} \subset \partial G$ then we have the mixed problem.

Many problems of mathematical physics lead one to consider the solution of boundary value problems for elliptic second order equations in unbounded domains and to study the behaviour of the solution at infinity, for instance stationary states, travelling waves, homogenization, boundary layer problems, Saint-Venant's principle and so on. Some problems in unbounded domains found applications in to mechanics of inhomogeneous media [9], models in astrophysics (Eddington's model), are used in the analytic theory of polytropic ball model of stellar structures (Lane-Ritter-Emden theory) and have numerous applications in natural sciences, e.g. in scalar field theory, in phase transition theory, in combustion theory, population dynamics [10], pseudo-plastic fluids $[6,8,12]$, and ecology models [14].

A number of mathematicians $[2,5,7,13,15,16]$ took the study of quasilinear elliptic problems in unbounded domains. The problem of the existence and nonexistence of positive solutions to a weak linear second-order divergence type elliptic equation in an unbounded cone-like domains was studied in [7].

Our aim is to establish the exact exponent of $(B V P)$ weak solutions decreasing rate. For this purpose in Section 2 we formulate and prove the comparison principle. Next, in Section 3, we show the boundedness of weak solutions to problem $(B V P)$. In section 4 we construct of the barrier function and study properties of solutions to the Sturm - Liouville boundary problem. Finally, in Section 5, we prove estimation of weak solutions for the problem $(B V P)$ near the infinity.

For $x=\left(x_{1}, \ldots, x_{n}\right)$ we introduce the cylindrical coordinates $\left(r, \omega, x^{\prime}\right)$, where $x^{\prime}=\left(x_{3}, \ldots, x_{n}\right), r=\sqrt{x_{1}^{2}+x_{2}^{2}}, \omega=\arctan \frac{x_{2}}{x_{1}}$. We assume that there exists $d \gg 1$ such that $G=G_{0} \cup G_{d}$, where $G_{0}$ is a bounded domain and:

$$
\begin{aligned}
G_{d} & =\left\{\left(r, \omega, x^{\prime}\right) \mid d<r<\infty, \omega \in\left(-\frac{\omega_{0}}{2}, \frac{\omega_{0}}{2}\right), x^{\prime} \in \mathbb{R}^{n-2}\right\}, 0<\omega_{0}<2 \pi \\
\partial G_{d} & =\Gamma_{d}^{+} \cup \Gamma_{d}^{-} \cup \Omega_{d}, \text { where } \\
\Omega_{d} & =\left\{\left(r, \omega, x^{\prime}\right) \mid r=d, \omega \in\left(-\frac{\omega_{0}}{2}, \frac{\omega_{0}}{2}\right), x^{\prime} \in \mathbb{R}^{n-2}\right\} ; \\
\Gamma_{d}^{ \pm} & =\left\{\left(r, \pm \frac{\omega_{0}}{2}, x^{\prime}\right) \mid r>d, x^{\prime} \in \mathbb{R}^{n-2}\right\} .
\end{aligned}
$$

Assumptions. The following conditions will be needed regarding to our 
BEST POSSIBLE ESTIMATES OF WEAK SOLUTIONS OF BOUNDARY VALUE PROBLEMS FOR QUASI-LINEAR ELLIPTIC EQUATIONS IN UNBOUNDED

problem :

(1) $f(x), g(x)$ are measurable functions such that:

$$
\begin{gathered}
f(x) \in L_{p}(G), g(x) \in L_{\alpha}(\partial G) ; \\
\frac{1}{p}<\frac{m}{n}-\frac{1}{t}, \quad \frac{1}{t}<\frac{m}{n}<1+\frac{1}{t}<m, \quad \alpha>\frac{n-1}{m-1-\frac{n}{t}} ;
\end{gathered}
$$

(2) $|g(x)| \leq g_{1} r^{\tau-m+1+(q+m-1) \lambda_{-}},|f(x)| \leq f_{1} r^{\tau-m+(q+m-1) \lambda_{-}}, \lambda_{-}<0$.

We denote by $\mathfrak{N}_{m, q, \tau}^{1}(G)$ the set of functions $u(x) \in C^{0}(\bar{G})$ having first weak derivatives with the finite integral $\int_{G}\left(r^{\tau}|u|^{q}|\nabla u|^{m}+r^{\tau-m}|u|^{q+m}\right) d x$ for $q \geq 0, m>1, \tau \leq m-2$.

Definition 1. A function $u(x)$ is said to be a weak solution of the problem (BVP) provided that $u(x) \in C^{0}(\bar{G}) \cap \mathfrak{N}_{m, q, \tau}^{1}(G)$ and satisfies the integral identity

$$
\begin{gathered}
\int_{G}\left\{r^{\tau}|u|^{q}|\nabla u|^{m-2} u_{x_{i}} \eta_{x_{i}}+\left(a_{0} r^{\tau-m} u|u|^{q+m-2}-\mu_{0} r^{\tau}|u|^{q-1}|\nabla u|^{m} \text { signu } \eta\right\} d x\right. \\
=\int_{G} f \eta d x+\int_{\partial G} \alpha(x)\left(g-\gamma(\omega) r^{\tau-m+1} u|u|^{q+m-2}\right) \eta d s
\end{gathered}
$$

for all functions $\eta(x) \in C^{0}(\bar{G}) \cap \mathfrak{N}_{m, q, \tau}^{1}(G)$ such that $\lim _{|x| \rightarrow \infty} \eta(x)=0$.

Lemma 1.1. Let $u(x)$ be a weak solution of $(B V P)$. For any function $\eta(x) \in C^{0}(\bar{G}) \cap \mathfrak{N}_{m, q, \tau}^{1}(G)$ with $\lim _{|x| \rightarrow \infty} \eta(x)=0$ the equality

$$
\begin{gathered}
\int_{G_{R}}\left\{r^{\tau}|u|^{q}|\nabla u|^{m-2} u_{x_{i}} \eta_{x_{i}}+\left(a_{0} r^{\tau-m} u|u|^{q+m-2}-\mu_{0} r^{\tau}|u|^{q-1}|\nabla u|^{m} \text { signu }\right) \eta d x\right. \\
=-\int_{\Omega_{R}} r^{\tau}|u|^{q}|\nabla u|^{m-2} u_{x_{i}} \eta \cos \left(r, x_{i}\right) d \Omega_{R}+\int_{G_{R}} f \eta d x \\
+\int_{\Gamma_{R}} \alpha(x)\left(g-\gamma(\omega) r^{\tau-m+1} u|u|^{q+m-2}\right) \eta d s .
\end{gathered}
$$

holds for a.e. $R>d \gg 1$.

Proof. The proof is analogous to the proof of Lemma 1.3 [18].

We shall consider the substitution

$$
u=v|v|^{\zeta-1} \text { with } \zeta=\frac{m-1}{q+m-1} .
$$


BEST POSSIBLE ESTIMATES OF WEAK SOLUTIONS OF BOUNDARY VALUE PROBLEMS FOR QUASI-LINEAR ELLIPTIC EQUATIONS IN UNBOUNDED

Then the identity $(I I)_{l o c}$ takes the following form

$$
\begin{gathered}
\int_{G_{R}}\left\{\zeta^{m-1} r^{\tau}|\nabla v|^{m-2} v_{x_{i}} \eta_{x_{i}}+\left(a_{0} r^{\tau-m} v|v|^{m-2}-\mu_{0} \zeta^{m} r^{\tau}|v|^{-1}|\nabla v|^{m} \operatorname{sign} v\right) \eta\right\} d x \\
=-\int_{\Omega_{R}} \zeta^{m-1} r^{\tau}|\nabla v|^{m-2} v_{x_{i}} \eta \cos \left(r, x_{i}\right) d \Omega_{R}+\int_{G_{R}} f \eta d x \\
\quad+\int_{\Gamma_{R}} \alpha(x)\left(g-\gamma(\omega) r^{\tau-m+1} v|v|^{m-2}\right) \eta d s
\end{gathered}
$$

for a.e. $R>d \gg 1, \quad v(x) \in C^{0}(\bar{G}) \cap \mathfrak{N}_{m, 0, \tau}^{1}(G)$ and any $\eta(x) \in C^{0}(\bar{G}) \cap$ $\mathfrak{N}_{m, 0, \tau}^{1}(G)$ with $\lim _{|x| \rightarrow \infty} \eta(x)=0$.

We need some auxiliary lemmas:

Lemma 1.2. Stampacchia's Lemma. (See Lemma 3.11 of [11]) Let $\varphi:\left[k_{0}, \infty\right) \rightarrow \mathbb{R}$ be a non-negative and non-increasing function which satisfies

$$
\varphi(h) \leq \frac{C}{(h-k)^{\alpha}}[\varphi(k)]^{\beta} \quad \text { for } \quad h>k>k_{0},
$$

where $C, \alpha, \beta$ are positive constants with $\beta>1$. Then $\varphi\left(k_{0}+d\right)=0$, where $d^{\alpha}=C \varphi^{\beta-1}\left(k_{0}\right) 2^{\frac{\alpha \beta}{\beta-1}}$.

Lemma 1.3. (see Lemma 2.1 in [3]). Let us consider the function

$$
\eta(x)= \begin{cases}e^{\varkappa x}-1 & x \geq 0 \\ -e^{-\varkappa x}+1 & x \leq 0\end{cases}
$$

where $\varkappa>0$. Let $a, b$ be positive constants, $m>1$. If $\varkappa>\frac{2 b}{a}+m$, then we have

$$
a \eta^{\prime}(x)-b|\eta(x)| \geq \frac{a}{2} e^{\varkappa x}, \quad \forall x \geq 0 .
$$

Moreover, there exist some $d \geq 0$ and $M>0$ such that

$$
\begin{gathered}
\eta(x) \leq M\left[\eta\left(\frac{x}{m}\right)\right]^{m}, \quad \eta^{\prime}(x) \leq M\left[\eta\left(\frac{x}{m}\right)\right]^{m}, \quad \forall x \geq d \\
|\eta(x)| \geq x, \quad \forall x \in \mathbb{R} .
\end{gathered}
$$

Proof. See the proof of Lemma 1.23 in [1]. 
BEST POSSIBLE ESTIMATES OF WEAK SOLUTIONS OF BOUNDARY VALUE PROBLEMS FOR QUASI-LINEAR ELLIPTIC EQUATIONS IN UNBOUNDED

Lemma 1.4. (see [4], Examples 1.5, 1.6, p. 29) Let $m^{\#}$ denote the number associated to $m$ by the relation

$$
\frac{1}{m^{\#}}=\frac{1}{m}\left(1+\frac{1}{t}\right)-\frac{1}{n}
$$

and assume that (1) holds. Then there exist positive constants $c_{1}, c_{2}$ (depending only on meas $D, n, m, t, \tau)$ such that

$$
\begin{gathered}
\int_{D} r^{\tau-m}|v|^{m} d x \leq c_{1} \int_{D} r^{\tau}|\nabla v|^{m} d x \\
\left(\int_{D}|v|^{m^{\#}} d x\right)^{\frac{m}{m \#}} \leq c_{2} \int_{D}\left(r^{\tau-m}|v|^{m}+r^{\tau}|\nabla v|^{m}\right) d x
\end{gathered}
$$

for any $v(x) \in \mathfrak{N}_{m, 0, \tau}^{1}(D)$.

Lemma 1.5. There exists a constant $c_{4}>0$ depending only on $n, m, t, D, \partial D$ such that for any $v(x) \in \mathfrak{N}_{m, 0, \tau}^{1}(D)$

$$
\left(\int_{\partial D}|v|^{\alpha^{*}} d s\right)^{\frac{1}{\alpha^{*}}} \leq c_{4}\left\{\int_{D}\left(r^{\tau-m}|v|^{m}+r^{\tau}|\nabla v|^{m}\right) d x\right\}^{\frac{1}{m}}, \quad \alpha^{*}=\frac{m(n-1)}{n-m+\frac{n}{t}} .
$$

This lemma is a simple implication of Theorem 3.9 of [11].

Corollary 1.6. From Lemmas 1.4 and 1.5

$$
\left(\int_{D}|v|^{m^{\#}} d x\right)^{\frac{m}{m^{\#}}}+\left(\int_{\partial D}|v|^{\alpha^{*}} d s\right)^{\frac{m}{\alpha^{*}}} \leq c_{3}\left\{\int_{D}\left(r^{\tau-m}|v|^{m}+r^{\tau}|\nabla v|^{m}\right) d x\right\}
$$

for any $v(x) \in \mathfrak{N}_{m, 0, \tau}^{1}(D)$, where $c_{3}>0$ depends on $n, m, t, D, \partial D$, $\left\|r^{m-\tau}\right\|_{L_{t}(D)},\left\|r^{-\tau}\right\|_{L_{t}(D)}$. 
BEST POSSIBLE ESTIMATES OF WEAK SOLUTIONS OF BOUNDARY VALUE PROBLEMS FOR QUASI-LINEAR ELLIPTIC EQUATIONS IN UNBOUNDED

\section{The comparison principle}

Taking into account $(\widehat{I I})_{l o c}$ we consider the second order quasi-linear degenerate operator $Q$ of the form

$$
\begin{aligned}
& Q(v, \eta) \equiv \int_{G_{d}}\left(\zeta^{m-1} r^{\tau}|\nabla v|^{m-2} v_{x_{i}} \eta_{x_{i}}+a_{0} r^{\tau-m} v|v|^{m-2} \eta(x)\right. \\
& \left.-\mu_{0} r^{\tau} \zeta^{m}|\nabla v|^{m}|v|^{-1} \eta(x) \cdot \operatorname{sign} v\right) d x+\int_{\Gamma_{d}} \alpha(x) \gamma(\omega) r^{\tau-m+1} v|v|^{m-2} \eta(x) d s \\
& +\int_{\Omega_{d}} r^{\tau} \zeta^{m-1}|\nabla v|^{m-2} v_{x_{i}} \eta(x) \cos \left(r, x_{i}\right) d \Omega_{d} \\
& -\int_{\Gamma_{d}} \alpha(x) g(x) \eta(x) d s-\int_{G_{d}} f(x) \eta(x) d x
\end{aligned}
$$

for $v(x) \in C^{0}\left(\overline{G_{d}}\right) \cap \mathfrak{N}_{m, 0, \tau}^{1}\left(G_{d}\right)$ and for all non-negative $\eta \in C^{0}\left(\overline{G_{d}}\right) \cap$ $\mathfrak{N}_{m, 0, \tau}^{1}\left(G_{d}\right)$ under the following assumptions: $f(x) \in L_{1}\left(G_{d}\right), g(x) \in L_{1}\left(\Gamma_{d}\right)$.

Proposition 2.1. Let $d \gg 1$, functions $u, w \in C^{0}\left(\overline{G_{d}}\right) \cap \mathfrak{N}_{m, 0, \tau}^{1}\left(G_{d}\right)$ and satisfy the inequality $Q(v, \eta) \leq Q(w, \eta)$ for all non-negative $\eta(x) \in C^{0}\left(\overline{G_{d}}\right) \cap$ $\mathfrak{N}_{m, 0, \tau}^{1}\left(G_{d}\right)$ and also the inequality $v(x) \leq w(x)$ holds on $\Omega_{d}$. Then $v(x) \leq$ $w(x)$ in $G_{d}$.

Proof. Let us define $z=v-w$ and $v^{t}=t v+(1-t) w, t \in[0,1]$. Then we have

$$
\begin{aligned}
& Q(v, \eta)-Q(w, \eta) \\
& =\int_{G_{d}}\left\{\zeta^{m-1} r^{\tau} \eta_{x_{i}} z_{x_{j}} \int_{0}^{1} \frac{\partial\left(\left|\nabla v^{t}\right|^{m-2} v_{x_{i}}^{t}\right)}{\partial v_{x_{j}}^{t}} d t+a_{0} r^{\tau-m} \eta z(x) \int_{0}^{1} \frac{\partial\left(v^{t}\left|v^{t}\right|^{m-2}\right)}{\partial v^{t}} d t\right. \\
& -\mu_{0} r^{\tau} \zeta^{m} \eta(x) z_{x_{i}} \int_{0}^{1} \frac{\partial\left(\left|\nabla v^{t}\right|^{m}\left|v^{t}\right|^{-1} \operatorname{sign} v^{t}\right)}{\partial v_{x_{i}}^{t}} d t \\
& \left.-\mu_{0} r^{\tau} \zeta^{m} \eta(x) z(x) \int_{0}^{1} \frac{\partial\left(\left|\nabla v^{t}\right|^{m}\left|v^{t}\right|^{-1} \operatorname{sign} v^{t}\right)}{\partial v^{t}} d t\right\} d x
\end{aligned}
$$


BEST POSSIBLE ESTIMATES OF WEAK SOLUTIONS OF BOUNDARY VALUE PROBLEMS FOR QUASI-LINEAR ELLIPTIC EQUATIONS IN UNBOUNDED

$$
\begin{aligned}
& +\int_{\Gamma_{d}} \alpha(x) \gamma(\omega) r^{\tau-m+1}\left(\int_{0}^{1} \frac{\partial\left(v^{t}\left|v^{t}\right|^{m-2}\right)}{\partial v^{t}} d t\right) z(x) \eta(x) d s \\
& +\zeta^{m-1} \int_{\Omega_{d}} r^{\tau}\left(\int_{0}^{1} \frac{\partial\left(\left|\nabla v^{t}\right|^{m-2} v_{x_{i}}^{t}\right)}{\partial v_{x_{j}}^{t}} d t\right) \cos \left(r, x_{i}\right) \cdot z_{x_{j}} \eta(x) d \Omega_{d} .
\end{aligned}
$$

In fact, for example

$$
\begin{aligned}
|\nabla v|^{m-2} v_{x_{i}}-|\nabla w|^{m-2} w_{x_{i}}=\left.\left|\nabla v^{t}\right|^{m-2} v_{x_{i}}^{t}\right|_{0} ^{1} & =\int_{0}^{1} \frac{\partial\left(\left|\nabla v^{t}\right|^{m-2} v_{x_{i}}^{t}\right)}{\partial v_{x_{j}}^{t}} \cdot \frac{\partial v_{x_{j}}^{t}}{\partial t} d t \\
& =z_{x_{j}} \int_{0}^{1} \frac{\partial\left(\left|\nabla v^{t}\right|^{m-2} v_{x_{i}}^{t}\right)}{\partial v_{x_{j}}^{t}} d t .
\end{aligned}
$$

Now we define following sets

$$
\begin{aligned}
& \left(G_{d}\right)^{+}:=\left\{x \in G_{d} \mid v(x)>w(x)\right\} \subset G_{d} \\
& \left(\Gamma_{d}\right)^{+}:=\left\{x \in \Gamma_{d} \mid v(x)>w(x)\right\} \subset \Gamma_{d}
\end{aligned}
$$

and assume that $\left(G_{d}\right)^{+} \neq \emptyset$. Let $k \geq 1$ be any odd number. As a test function in (10) we choose $\eta=\max \left\{(v-w)^{k}, 0\right\}$. In virtue of the fact that

$$
\int_{0}^{1} \frac{\partial\left(v^{t}\left|v^{t}\right|^{m-2}\right)}{\partial v^{t}} d t=(m-1) \int_{0}^{1}\left|v^{t}\right|^{m-2} d t>0
$$

and because $\left.\eta\right|_{\Omega_{d}}=0$, we obtain from (10) that

$$
\begin{gathered}
\int_{\left(G_{d}\right)^{+}}\left\{k \zeta^{m-1} r^{\tau} z^{k-1} z_{x_{i}} z_{x_{j}} \int_{0}^{1} \frac{\partial\left(\left|\nabla v^{t}\right|^{m-2} v_{x_{i}}^{t}\right)}{\partial v_{x_{j}}^{t}} d t\right. \\
-\mu_{0} r^{\tau} \zeta^{m} z^{k} z_{x_{i}} \int_{0}^{1} \frac{\partial\left(\left|\nabla v^{t}\right|^{m}\left(v^{t}\right)^{-1}\right)}{\partial v_{x_{i}}^{t}} d t \\
\left.-\mu_{0} r^{\tau} \zeta^{m} z^{k+1} v^{t} \int_{0}^{1} \frac{\partial\left(\left|\nabla v^{t}\right|^{m}\left(v^{t}\right)^{-1}\right)}{\partial v^{t}} d t\right\} d x \leq 0 .
\end{gathered}
$$


BEST POSSIBLE ESTIMATES OF WEAK SOLUTIONS OF BOUNDARY VALUE PROBLEMS FOR QUASI-LINEAR ELLIPTIC EQUATIONS IN UNBOUNDED DOMAINS

Now, because :

$$
\begin{gathered}
\frac{\partial\left(\left|\nabla v^{t}\right|^{m-2} v_{x_{i}}^{t}\right)}{\partial v_{x_{j}}^{t}} z_{x_{i}} z_{x_{j}}=\left(\left|\nabla v^{t}\right|^{m-2} \delta_{i}^{j}+(m-2)\left|\nabla v^{t}\right|^{m-4} v_{x_{i}}^{t} v_{x_{j}}^{t}\right) z_{x_{i}} z_{x_{j}} \\
\geq \gamma_{m}\left|\nabla v^{t}\right|^{m-2}|\nabla z|^{2}, \quad \text { where } \gamma_{m}= \begin{cases}1, & m \geq 2 ; \\
m-1, & 1<m \leq 2 ;\end{cases} \\
\frac{\partial\left(\left|\nabla v^{t}\right|^{m}\left(v^{t}\right)^{-1}\right)}{\partial v^{t}}=-\frac{\left|\nabla v^{t}\right|^{m}}{\left(v^{t}\right)^{2}} ; \frac{\partial\left(\left|\nabla v^{t}\right|^{m}\left(v^{t}\right)^{-1}\right)}{\partial v_{x_{i}}^{t}}=m\left|\nabla v^{t}\right|^{m-2} v_{x_{j}}^{t}\left(v^{t}\right)^{-1},
\end{gathered}
$$

then (11) yields

$$
\begin{aligned}
\int_{\left(G_{d}\right)^{+}} r^{\tau}\left\{k \gamma_{m} \zeta^{m-1} z^{k-1}\left(\int_{0}^{1}\left|\nabla v^{t}\right|^{m-2} d t\right)|\nabla z|^{2}\right. \\
\left.+\mu_{0} \zeta^{m} z^{k+1}\left(\int_{0}^{1}\left|v^{t}\right|^{-2}\left|\nabla v^{t}\right|^{m} d t\right)\right\} d x \\
\leq \int_{\left(G_{d}\right)^{+}} m \mu_{0} r^{\tau} \zeta^{m} z^{k}\left(\int_{0}^{1}\left|v^{t}\right|^{-1}\left|\nabla v^{t}\right|^{m-1} d t\right)|\nabla z| d x
\end{aligned}
$$

On account of the Cauchy inequality with $\varepsilon=2$

$$
\begin{aligned}
m z^{k}|\nabla z|\left|v^{t}\right|^{-1}\left|\nabla v^{t}\right|^{m-1} & =\left(\left|v^{t}\right|^{-1} z^{\frac{k+1}{2}}\left|\nabla v^{t}\right|^{\frac{m}{2}}\right) \cdot\left(m z^{\frac{k-1}{2}}|\nabla z|\left|\nabla v^{t}\right|^{\frac{m}{2}-1}\right) \\
& \leq\left|v^{t}\right|^{-2} z^{k+1}\left|\nabla v^{t}\right|^{m}+\frac{1}{4} m^{2} z^{k-1}|\nabla z|^{2}\left|\nabla v^{t}\right|^{m-2}
\end{aligned}
$$

we obtain from (12) that

$$
\left(k \gamma_{m}-\frac{1}{4} m^{2} \mu_{0} \zeta\right) \int_{\left(G_{d}\right)^{+}} r^{\tau} z^{k-1}|\nabla z|^{2}\left(\int_{0}^{1}\left|\nabla v^{t}\right|^{m-2} d t\right) d x \leq 0 .
$$

Thus, choosing the odd number $k \geq \max \left(1, \frac{m^{2} \mu_{0} \zeta}{2 \gamma_{m}}\right)$, in view of $z(x) \equiv 0$ on $\partial\left(G_{d}\right)^{+}$, we get $z(x) \equiv 0$ in $\left(G_{d}\right)^{+}$. We have arrived to a contradiction to our definition of the set $\left(G_{d}\right)^{+}$. By this fact, the proposition is proved.

\section{The boundedness of weak solutions}

In this section we consider one of the possible cases of deriving $L_{\infty}(G)$ a priori estimate of the weak solution to problem $(B V P)$. 
BEST POSSIBLE ESTIMATES OF WEAK SOLUTIONS OF BOUNDARY VALUE PROBLEMS FOR QUASI-LINEAR ELLIPTIC EQUATIONS IN UNBOUNDED DOMAINS

We first observe that there exists $R^{*}>1$ such that $|u(x)|<1$ for all $x \in G_{R^{*}}$. We denote $G^{*} \equiv G \backslash G_{R^{*}}$ and introduce the set $A(k)=$ $\left\{x \in \overline{G^{*}},|u(x)|>k\right\}$. We shall use the notation: $(|u|-k)_{+}:=\max (|u|-k, 0)$.

Theorem 3.1. Let $u(x)$ be a weak solution of (BVP) and assumption (1) hold. Then there exists the constant $M_{0}>0$ depending only on $\|f\|_{L_{p}(G)}$, $\|g\|_{L_{\alpha}(\partial G)},\left\|r^{m-\tau}\right\|_{L_{t}\left(G^{*}\right)},\left\|r^{-\tau}\right\|_{L_{t}\left(G^{*}\right)}, n, m, t$, meas $G^{*}$, meas $\partial G^{*}$, such that $\|u\|_{L_{\infty}(G)} \leq M_{0}$.

By setting $\eta\left((|u|-k)_{+}\right) \chi_{A(k)} \cdot \operatorname{sign} u$ as test function in (II), where $\eta$ is defined by Lemma $1.3, \chi_{A(k)}$ is the characteristic function of the set $A(k)$ and $k \geq k_{0}$ (without loss of generality we can assume $k_{0} \geq 1$ ) we get:

$$
\begin{aligned}
& \int_{A(k)}\left\{r^{\tau}|u|^{q}|\nabla u|^{m} \eta^{\prime}(|u|-k)+a_{0} r^{\tau-m}|u|^{q+m-1} \eta(|u|-k)\right\} d x \\
& \quad+\int_{\partial G \cap A(k)} \alpha(x) \gamma(\omega) r^{\tau-m+1}|u|^{q+m-1} \eta(|u|-k) d s \\
& \quad \leq \int_{A(k)}\left\{\mu_{0} r^{\tau}|u|^{q-1}|\nabla u|^{m} \eta(|u|-k)+|f(x)| \eta(|u|-k)\right\} d x \\
& +\int_{\partial G \cap A(k)} \alpha(x)|g(x)| \eta(|u|-k) d s .
\end{aligned}
$$

Now we define the function $w_{k}(x):=\eta\left(\frac{|u|-k}{m}\right)$. By (4), from Lemma 1.3, we have that

$$
\begin{gathered}
\int_{\partial G \cap A(k)} \alpha(x)|g(x)| \eta(|u|-k) d s \leq M \int_{\partial G \cap A(k+d)} \alpha(x)|g(x)|\left|w_{k}\right|^{m} d s \\
+\mathrm{e}^{\varkappa d} \int_{\partial G \cap\{A(k) \backslash A(k+d)\}} \alpha(x)|g(x)| d s \quad \forall d>0 .
\end{gathered}
$$

Along similiar lines we can estimate the integral $\int_{A(k)}|f(x)| \eta(|u|-k) d x$. 
BEST POSSIBLE ESTIMATES OF WEAK SOLUTIONS OF BOUNDARY VALUE PROBLEMS FOR QUASI-LINEAR ELLIPTIC EQUATIONS IN UNBOUNDED

Now we apply Lemma 1.5. In virtue of the Hölder inequality and (7) we get

$$
\begin{gathered}
\int_{\partial G \cap A(k+d)}|g(x)|\left|w_{k}\right|^{m} d s \leq\left(\int_{\partial G \cap A(k+d)}\left|w_{k}\right|^{\alpha^{*}} d s\right)^{\frac{m}{\alpha^{*}}}\|\alpha(x) g(x)\|_{L \frac{n-1}{m-1-\frac{n}{t}}(\partial G)} \\
\leq c_{4}\|\alpha(x) g(x)\|_{\frac{n-1}{m-1-\frac{n}{t}(\partial G)}} \int_{A(k)}\left(r^{\tau}\left|\nabla w_{k}\right|^{m}+r^{\tau-m}\left|w_{k}\right|^{m}\right) d x
\end{gathered}
$$

Then, because $|u|^{q-1} \leq|u|^{q} \cdot k_{0}^{-1}$, from (13) and (14) it follows that

$$
\begin{aligned}
& \int_{A(k)} r^{\tau}|u|^{q}|\nabla u|^{m}\left(\eta^{\prime}(|u|-k)-\mu_{0} k_{0}^{-1} \eta(|u|-k)\right) d x \\
& \leq M c_{4}\|\alpha(x) g(x)\|_{L \frac{n-1}{m-1-\frac{n}{t}(\partial G)}} \cdot \int_{A(k)}\left(r^{\tau}\left|\nabla w_{k}\right|^{m}+r^{\tau-m}\left|w_{k}\right|^{m}\right) d x \\
& +c_{5} M \int_{A(k+d)}|f|\left|w_{k}\right|^{m} d x+c_{6} \mathrm{e}^{\varkappa d}\left\{\int_{\{A(k) \backslash A(k+d)\}}|f| d x+\int_{\partial G \cap A(k)}|g| d s\right\} .
\end{aligned}
$$

By the definition of $\eta(x)$ and $w_{k}(x)$ we get $e^{\varkappa(|u|-k)+|\nabla u|^{m}}=\left(\frac{m}{\varkappa}\right)^{m}\left|\nabla w_{k}\right|^{m}$ for $\varkappa>0$. Therefore, by the choice of $\varkappa>m+\frac{2 \mu_{0}}{k_{0}}$ according to Lemma 1.3, using (3), from (15) we obtain

$$
\begin{aligned}
& \frac{1}{2} k_{0}^{q}\left(\frac{m}{\varkappa}\right)^{m} \int_{A(k)} r^{\tau}\left|\nabla w_{k}\right|^{m} d x \\
& \leq c_{4} M\|\alpha(x) g\|_{L} \frac{n-1}{m-1-\frac{n}{t}(\Gamma)} \int_{A(k)}\left(r^{\tau}\left|\nabla w_{k}\right|^{m}+r^{\tau-m}\left|w_{k}\right|^{m}\right) d x \\
& +c_{5} M \int_{A(k+d)}|f|\left|w_{k}\right|^{m} d x+c_{6} \mathrm{e}^{\varkappa d}\left\{\int_{\{A(k) \backslash A(k+d)\}}|f| d x+\int_{\partial G \cap A(k)}|g| d s\right\} .
\end{aligned}
$$


BEST POSSIBLE ESTIMATES OF WEAK SOLUTIONS OF BOUNDARY VALUE PROBLEMS FOR QUASI-LINEAR ELLIPTIC EQUATIONS IN UNBOUNDED DOMAINS

Now, by (7), the inequality above gives

$$
\begin{aligned}
& \left(k_{0}^{q}-c_{7}\right) \int_{A(k)} r^{\tau}\left|\nabla w_{k}\right|^{m} d x \\
& \leq c_{8} \int_{A(k+d)}|f(x)|\left|w_{k}\right|^{m} d x+c_{9}\left\{\int_{A(k)}|f(x)| d x+\int_{\partial G \cap A(k)}|g(x)| d s\right\},
\end{aligned}
$$

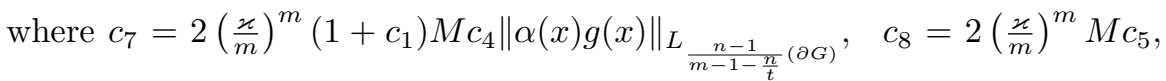
$c_{9}=2\left(\frac{\varkappa}{m}\right)^{m} c_{6} \mathrm{e}^{\varkappa d}$.

Using the Hölder inequality with exponents $p$ and $p^{\prime}$ for the first integral on the right hand side, we obtain:

$$
\int_{A(k+d)}|f(x)|\left|w_{k}\right|^{m} d x \leq\|f(x)\|_{L_{p}(G)}\left(\int_{A(k)}\left|w_{k}\right|^{m p^{\prime}} d x\right)^{\frac{1}{p^{\prime}}} .
$$

From the inequality $\frac{1}{p}<\frac{m}{n}-\frac{1}{t}$ it follows that $m p^{\prime}<m^{\#}$, where $m^{\#}$ is defined by (6). Let $j$ be a real number such that $m p^{\prime}<j<m^{\#}$. In virtue of the interpolation inequality we get

$$
\left(\int_{A(k)}\left|w_{k}\right|^{m p^{\prime}} d x\right)^{\frac{1}{p^{\prime}}} \leq\left(\int_{A(k)}\left|w_{k}\right|^{m} d x\right)^{\theta} \cdot\left(\int_{A(k)}\left|w_{k}\right|^{j} d x\right)^{\frac{(1-\theta) m}{j}}
$$

with $\theta \in(0,1)$, which is defined by the equality $\frac{1}{m p^{\prime}}=\frac{\theta}{m}+\frac{1-\theta}{j}$. On strength of the Hölder inequality with exponents $\frac{m^{\#}}{j}$ and $\frac{m^{\#}}{m^{\#}-j}$, from (17) we have :

$$
\int_{A(k+d)}|f(x)|\left|w_{k}\right|^{m} d x \leq c_{10}\left(\int_{A(k)}\left|w_{k}\right|^{m} d x\right)^{\theta} \cdot\left(\int_{A(k)}\left|w_{k}\right|^{m^{\#}} d x\right)^{\frac{(1-\theta) m}{m \#}}
$$

where $c_{10}=\|f(x)\|_{L_{p}(G)}(\text { meas } A(k))^{\frac{m\left(m_{\#}^{\#}-j\right)(1-\theta)}{j m \#}}$. Using the Young inequality with exponents $\frac{1}{\theta}$ and $\frac{1}{1-\theta}$, we obtain

$$
\int_{A(k+d)}|f|\left|w_{k}\right|^{m} d x \leq \frac{c_{11}}{\varepsilon^{\frac{1}{\theta}}} \int_{A(k)}\left|w_{k}\right|^{m} d x+\varepsilon^{\frac{1}{1-\theta}}(1-\theta)\left(\int_{A(k)}\left|w_{k}\right|^{m^{\#}} d x\right)^{\frac{m}{m \#}}
$$


BEST POSSIBLE ESTIMATES OF WEAK SOLUTIONS OF BOUNDARY VALUE PROBLEMS FOR QUASI-LINEAR ELLIPTIC EQUATIONS IN UNBOUNDED

for all $\varepsilon>0$, where $c_{11}=\theta\|f(x)\|_{L_{p}(G)}^{\frac{1}{\theta}}(\operatorname{meas} A(k))^{\frac{m(m \#-j)(1-\theta)}{j m \#}}$.

Now (18) and (16) yield:

$$
\begin{aligned}
& \left(k_{0}^{q}-c_{7}\right) \int_{A(k)} r^{\tau}\left|\nabla w_{k}\right|^{m} d x \\
& \leq c_{12} \varepsilon^{-\frac{1}{\theta}} \int_{A(k)}\left|w_{k}\right|^{m} d x+c_{9}\left\{\int_{A(k)}|f(x)| d x+\int_{\partial G \cap A(k)}|g(x)| d s\right\} \\
& +c_{13} \varepsilon^{\frac{1}{1-\theta}}\left(\int_{A(k)}\left|w_{k}\right|^{m^{\#}} d x\right)^{\frac{m}{m^{\#}}}, \forall \varepsilon>0 .
\end{aligned}
$$

Further, by (7), we obtain:

$$
\int_{A(k)}\left|w_{k}\right|^{m} d x=\int_{A(k)} r^{\tau-m}\left|w_{k}\right|^{m} r^{m-\tau} d x \leq c_{1}(\operatorname{diam} A(k)) \int_{A(k)} r^{\tau}\left|\nabla w_{k}\right|^{m} d x .
$$

By the two inequalities above, we have

$$
\begin{gathered}
\left(k_{0}^{q}-c_{7}-c_{14} \varepsilon^{-\frac{1}{\theta}}\right) \int_{A(k)} r^{\tau}\left|\nabla w_{k}\right|^{m} d x \leq c_{9}\left\{\int_{A(k)}|f(x)| d x+\int_{\partial G \cap A(k)}|g(x)| d s\right\} \\
+c_{13} \varepsilon^{\frac{1}{1-\theta}}\left(\int_{A(k)}\left|w_{k}\right|^{m^{\#}} d x\right)^{\frac{m}{m^{\#}}}, \forall \varepsilon>0, \forall k \geq k_{0} .
\end{gathered}
$$

Let us choose now

$$
\left\{\begin{array}{l}
c_{14} \varepsilon^{-\frac{1}{\theta}}=\frac{1}{2} k_{0}^{q} \Rightarrow \varepsilon=\left(2 c_{14}\right)^{\theta} k_{0}^{-q \theta} \\
k_{0}^{q} \geq 4 c_{7}
\end{array}\right.
$$


BEST POSSIBLE ESTIMATES OF WEAK SOLUTIONS OF BOUNDARY VALUE PROBLEMS FOR QUASI-LINEAR ELLIPTIC EQUATIONS IN UNBOUNDED

Then, by virtue of inequality (8), we have

$$
\begin{aligned}
\left(\frac{k_{0}^{q}}{4 c_{2}}-c_{13} \varepsilon^{\frac{1}{1-\theta}}\right) & \cdot\left\{\left(\int_{A(k)}\left|w_{k}\right|^{m^{\#}} d x\right)^{\frac{m}{m_{\#}}}+\left(\int_{\partial G \cap A(k)}\left|w_{k}\right|^{\alpha^{*}} d s\right)^{\frac{m}{\alpha^{*}}}\right\} \\
& \leq c_{9}\left\{\int_{A(k)}|f(x)| d x+\int_{\partial G \cap A(k)}|g(x)| d s\right\}, \forall k \geq k_{0} .
\end{aligned}
$$

Now, if $\frac{k_{0}^{q}}{8 c_{2}} \geq c_{13} \varepsilon^{\frac{1}{1-\theta}}$, so by (19) we choose

$$
k_{0} \geq \max \left\{1 ;\left(8 c_{2} c_{13}\right)^{\frac{1-\theta}{q}}\left(2 c_{14}\right)^{\frac{\theta}{q}} ;\left(4 c_{7}\right)^{\frac{1}{q}}\right\}
$$

then from (20) we obtain

$$
\begin{aligned}
&\left(\int_{A(k)}\left|w_{k}\right|^{m^{\#}} d x\right)^{\frac{m}{m \#}}+\left(\int_{\partial G \cap A(k)}\left|w_{k}\right|^{\alpha^{*}} d s\right)^{\frac{m}{\alpha^{*}}} \\
& \leq c_{15}\left\{\int_{A(k)}|f(x)| d x+\int_{\partial G \cap A(k)}|g(x)| d s\right\} .
\end{aligned}
$$

Let now $l>k>k_{0}$. By inequality (5) and because $\left|w_{k}\right| \geq \frac{1}{m}(|u|-$ $k)_{+}$, we have $\int_{A(l)}\left|w_{k}\right|^{m^{\#}} d x \geq\left(\frac{l-k}{m}\right)^{m^{\#}} \operatorname{meas} A(l), \int_{\partial G \cap A(l)}\left|w_{k}\right|^{\alpha^{*}} d s \geq\left(\frac{l-k}{m}\right)^{\alpha^{*}}$ - meas $(\partial G \cap A(l))$. We also observe that

$$
\begin{gathered}
\int_{A(k)}|f(x)| d x \leq\|f\|_{L_{p}(G)} \operatorname{meas}^{1-\frac{1}{p}} A(k) ; \\
\int_{\partial G \cap A(k)}|g(x)| d s \leq\|g\|_{L_{\alpha}(\partial G)} \operatorname{meas}^{\frac{1}{\alpha^{\prime}}}(\partial G \cap A(k)), \quad \frac{1}{\alpha}+\frac{1}{\alpha^{\prime}}=1 .
\end{gathered}
$$


From (21), by the Jensen inequality, we get

$$
\begin{aligned}
& \operatorname{meas} A(l)+\operatorname{meas}^{\frac{m^{\#}}{\alpha^{*}}}(\partial G \cap A(l)) \\
& \leq\left(\frac{m}{l-k}\right)^{m^{\#}}\left\{\int_{A(k)}\left|w_{k}\right|^{m^{\#}} d x+\left(\int_{\partial G \cap A(k)}\left|w_{k}\right|^{\alpha^{*}} d s\right)^{\frac{m}{\alpha^{*}}}\right\} \\
& \leq 2^{\frac{m^{\#}}{m}-1}\left(\frac{m}{l-k}\right)^{m^{\#}} c_{15}^{\frac{m^{\#}}{m}}\left(\|f\|_{L_{p}(G)}+\|g\|_{L_{\alpha}(\partial G)}\right)^{\frac{m^{\#}}{m}} \\
& \cdot\left(\text { meas }^{\frac{m^{\#}}{m}}\left(1-\frac{1}{p}\right) A(k)+\operatorname{meas}^{\frac{m^{\#}}{m \alpha^{\prime}}}(\partial G \cap A(k))\right) .
\end{aligned}
$$

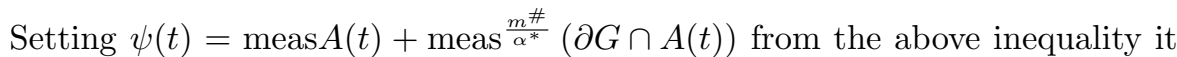
follows that

$$
\psi(l) \leq c_{16}\left(\frac{m}{l-k}\right)^{m^{\#}}\left\{[\psi(k)]^{\frac{m^{\#}}{m}\left(1-\frac{1}{p}\right)}+[\psi(k)]^{\frac{\alpha^{*}}{m \alpha^{\prime}}}\right\} .
$$

Notice that from assumption (1), definition of $m^{\#}$ and $\alpha^{*}$, we conclude that $\gamma=\min \left\{\frac{m^{\#}}{m}\left(1-\frac{1}{p}\right) ; \frac{\alpha^{*}}{m \alpha^{\prime}}\right\}>1$. In this way $(22)$ yields

$$
\psi(l) \leq \frac{c_{17}}{(l-k)^{m^{\#}}} \psi^{\gamma}(k) \quad \forall l>k \geq k_{0}
$$

and therefore we have, because of the Stampacchia Lemma, that $\psi\left(k_{0}+\delta\right)=0$ with $\delta$ depending only on quantities in the formulation of Theorem 3.1. This fact means that $|u(x)|<k_{0}+\delta$ for almost all $x \in G^{*}$. By the definition of $R^{*}$ it is obvious that $|u(x)|<k_{0}+\delta$ for almost all $x \in G$. Theorem 3.1 is proved.

\section{Construction of the barrier function}

In this section, for an $n$ - dimensional unbounded domain we consider the following elliptic problem for the model equation:

$$
\left\{\begin{array}{l}
-\frac{d}{d x_{i}}\left(r^{\tau}|w|^{q}|\nabla w|^{m-2} w_{x_{i}}\right)+a_{0} r^{\tau-m} w|w|^{q+m-2} \\
-\mu r^{\tau} w|w|^{q-2}|\nabla w|^{m}=0, \quad x \in G_{d} \\
\alpha(x)|w|^{q}|\nabla w|^{m-2} \frac{\partial w}{\partial \vec{n}}+\gamma r^{1-m} w|w|^{q+m-2}=0, \quad x \in \partial G_{d} ; \\
\lim _{|x| \rightarrow \infty} w(x)=0,
\end{array}\right.
$$


BEST POSSIBLE ESTIMATES OF WEAK SOLUTIONS OF BOUNDARY VALUE PROBLEMS FOR QUASI-LINEAR ELLIPTIC EQUATIONS IN UNBOUNDED

where $\gamma=\left\{\begin{array}{l}\gamma_{+}<\gamma\left(\frac{\omega_{0}}{2}\right), \quad x \in \Gamma_{d}^{+}, \\ \gamma_{-}<\gamma\left(-\frac{\omega_{0}}{2}\right), x \in \Gamma_{d}^{-} .\end{array} \quad a_{0} \geq 0, \mu>\mu_{0}, q \geq 0, m>1, \tau \leq m-2\right.$.

We shall seek a solution of the problem $(M V P)$ in the form

$$
w(x)=r^{\lambda_{-}} \Theta(\omega), \quad \omega \in\left[-\frac{\omega_{0}}{2}, \frac{\omega_{0}}{2}\right], \quad \lambda_{-}<0 .
$$

Substituting $w(x)$ in $(M V P)$ and calculating in the cylindrical coordinates, we get the following Sturm-Liouville boundary problem for the function $\Theta(\omega)$ :

$$
\left\{\begin{array}{l}
\frac{d}{d \omega}\left[\left(\lambda_{-}^{2} \Theta^{2}+\Theta^{\prime 2}\right)^{\frac{m-2}{2}}|\Theta|^{q} \Theta^{\prime}\right] \\
+\lambda_{-}\left[\lambda_{-}(q+m-1)-m+2+\tau\right] \Theta|\Theta|^{q}\left(\lambda_{-}^{2} \Theta^{2}+\Theta^{\prime 2}\right)^{\frac{m-2}{2}} \\
=a_{0} \Theta|\Theta|^{q+m-2}-\mu \Theta|\Theta|^{q-2}\left(\lambda_{-}^{2} \Theta^{2}+\Theta^{\prime 2}\right)^{\frac{m}{2}}, \quad \omega \in\left(-\frac{\omega_{0}}{2}, \frac{\omega_{0}}{2}\right), \\
\pm\left.\alpha(x)|\Theta|^{q}\left(\lambda_{-}^{2} \Theta^{2}+\Theta^{\prime 2}\right)^{\frac{m-2}{2}} \Theta^{\prime}\right|_{\omega= \pm \frac{\omega_{0}}{2}}+\left.\gamma_{ \pm} \Theta|\Theta|^{q+m-2}\right|_{\omega= \pm \frac{\omega_{0}}{2}}=0 .
\end{array}\right.
$$

Performing the function change $w=z|z|^{\zeta-1}$ with $\zeta=\frac{m-1}{q+m-1}$ we get the problem for the barrier function $z$ :

$$
\left\{\begin{aligned}
-\frac{d}{d x_{i}}\left(r^{\tau}|\nabla z|^{m-2} z_{x_{i}}\right)+\bar{a}_{0} r^{\tau-m} z|z|^{m-2} & \\
-\bar{\mu} r^{\tau} z^{-1}|\nabla z|^{m}=0, & x \in G_{d} ; \\
\alpha(x) \zeta^{m-1}|\nabla z|^{m-2} \frac{\partial z}{\partial \vec{n}}+\gamma r^{1-m} z|z|^{m-2}=0, & x \in \partial G_{d} ; \\
\lim _{|x| \rightarrow \infty} z(x)=0, &
\end{aligned}\right.
$$

where $\bar{a}_{0}=a_{0} \zeta^{1-m}, \bar{\mu}=\mu \zeta$.

For the solution $z$ of the problem $(\overline{M V P})$ we have the representation

$$
z(x)=r^{\bar{\lambda}_{-}} \psi(\omega), \psi(\omega)=\Theta(\omega)|\Theta(\omega)|^{\frac{1-\zeta}{\zeta}}, \quad \bar{\lambda}_{-}=\frac{\lambda_{-}}{\zeta}, \frac{\psi^{\prime}(\omega)}{\psi(\omega)}=\frac{\Theta^{\prime}(\omega)}{\zeta \Theta(\omega)} .
$$

Substituting the function $(23)$ in $(\overline{M V P})$ and calculating in the polar coordinates, we get the following Sturm-Liouville boundary value problem for the 
BEST POSSIBLE ESTIMATES OF WEAK SOLUTIONS OF BOUNDARY VALUE PROBLEMS FOR QUASI-LINEAR ELLIPTIC EQUATIONS IN UNBOUNDED

function $\psi(\omega)$ :

$$
\left\{\begin{array}{l}
\frac{d}{d \omega}\left[\left(\bar{\lambda}_{-}^{2} \psi^{2}+\psi^{\prime 2}\right)^{\frac{m-2}{2}} \psi^{\prime}\right] \\
+\bar{\lambda}_{-}\left[\bar{\lambda}_{-}(m-1)-m+2+\tau\right] \psi\left(\bar{\lambda}_{-}^{2} \psi^{2}+\psi^{\prime 2}\right)^{\frac{m-2}{2}} \\
=\bar{a}_{0} \psi|\psi|^{m-2}-\bar{\mu} \psi^{-1}\left(\bar{\lambda}_{-}^{2} \psi^{2}+\psi^{\prime 2}\right)^{\frac{m}{2}}, \quad \omega \in\left(-\frac{\omega_{0}}{2}, \frac{\omega_{0}}{2}\right) \\
\pm\left.\alpha(x)\left(\bar{\lambda}_{-}^{2} \psi^{2}+\psi^{\prime 2}\right)^{\frac{m-2}{2}} \psi^{\prime}\right|_{\omega= \pm \frac{\omega_{0}}{2}}+\left.\gamma_{ \pm} \psi|\psi|^{m-2}\right|_{\omega= \pm \frac{\omega_{0}}{2}}=0
\end{array}\right.
$$

The problem is a nonlinear eigenvalue problem. Now we want to study properties of eigenfunctions $\psi(\omega)$.

Lemma 4.1. Let $\lambda_{-}<0$ be an eigenvalue and $\psi(\omega)$ be the associated eigenfunction of $(\overline{S t L})$. Suppose that following inequalities are satisfied

$$
\begin{aligned}
& \left|\lambda_{-}\right| \geq\left(\frac{a_{0}}{\mu+\frac{m-2}{4 \zeta}}\right)^{\frac{1}{m}} ; \mu>\frac{2-m}{4 \zeta} ; \zeta=\frac{m-1}{q+m-1}, \quad \text { if } 1<m<2 ; \\
& \left\{\begin{array}{lc}
\left|\lambda_{-}\right|>\left(\frac{a_{0}}{\mu}\right)^{\frac{1}{m}} \text { for } \mu>0, & \text { if } m \geq 2 \\
(q+m-1)\left|\lambda_{-}\right|^{m}+(m-2-\tau)\left|\lambda_{-}\right|^{m-1} \geq a_{0} \text { for } \mu=0 . &
\end{array}\right.
\end{aligned}
$$

Then $\psi \psi^{\prime \prime} \leq 0$.

Proof. We rewrite the $(\overline{S t L})$ equation in the form

$$
\begin{aligned}
& -\psi \psi^{\prime \prime}\left\{\bar{\lambda}_{-}^{2} \psi^{2}+(m-1) \psi^{\prime 2}\right\}\left(\bar{\lambda}_{-}^{2} \psi^{2}+\psi^{\prime 2}\right)^{\frac{m-4}{2}} \\
& =\bar{\mu}\left(\bar{\lambda}_{-}^{2} \psi^{2}+\psi^{\prime 2}\right)^{\frac{m}{2}}-\bar{a}_{0}|\psi|^{m}+(m-2) \bar{\lambda}_{-}^{2} \psi^{2} \psi^{\prime 2}\left(\bar{\lambda}_{-}^{2} \psi^{2}+\psi^{\prime 2}\right)^{\frac{m-4}{2}} \\
& +\bar{\lambda}_{-}\left[\bar{\lambda}_{-}(m-1)+2+\tau-m\right]\left(\bar{\lambda}_{-}^{2} \psi^{2}+\psi^{\prime 2}\right)^{\frac{m-2}{2}} \psi^{2} \equiv j(\psi) .
\end{aligned}
$$

At first we consider the case when $1<m<2$. By the Cauchy inequality

$$
-\bar{\lambda}_{-}|\psi|\left|\psi^{\prime}\right| \leq \frac{1}{2}\left(\bar{\lambda}_{-}^{2} \psi^{2}+\psi^{\prime 2}\right) \Rightarrow(m-2) \bar{\lambda}_{-}^{2} \psi^{2} \psi^{\prime 2} \geq \frac{m-2}{4}\left(\bar{\lambda}_{-}^{2} \psi^{2}+\psi^{\prime 2}\right)^{2} .
$$

Simple observation gives

$$
\bar{a}_{0}|\psi|^{m}=\frac{\bar{a}_{0}}{\left|\bar{\lambda}_{-}\right|^{m}}\left(\bar{\lambda}_{-}^{2} \psi^{2}\right)^{\frac{m}{2}} \leq \frac{\bar{a}_{0}}{\left|\bar{\lambda}_{-}\right|^{m}}\left(\bar{\lambda}_{-}^{2} \psi^{2}+\psi^{\prime 2}\right)^{\frac{m}{2}} .
$$


BEST POSSIBLE ESTIMATES OF WEAK SOLUTIONS OF BOUNDARY VALUE PROBLEMS FOR QUASI-LINEAR ELLIPTIC EQUATIONS IN UNBOUNDED

Hence $j(\psi) \geq\left(\bar{\mu}+\frac{m-2}{4}-\frac{\bar{a}_{0}}{\left|\bar{\lambda}_{-}\right|^{m}}\right)\left(\bar{\lambda}_{-}^{2} \psi^{2}+\psi^{\prime 2}\right)^{\frac{m}{2}}$, in virtue of $2+\tau-m \leq 0$. If (24) holds, then the required statement follows.

Let us consider the case when $m \geq 2$. If $\mu>0$, then from (26)-(27) we obtain

$$
\begin{aligned}
& -\psi \psi^{\prime \prime}\left\{\bar{\lambda}_{-}^{2} \psi^{2}+(m-1) \psi^{\prime 2}\right\} \\
& \geq\left(\bar{\mu}-\frac{\bar{a}_{0}}{\left|\bar{\lambda}_{-}\right|^{m}}\right)\left(\bar{\lambda}_{-}^{2} \psi^{2}+\psi^{\prime 2}\right)^{2}+(m-2) \bar{\lambda}_{-}^{2} \psi^{2} \psi^{\prime 2} \\
& +\bar{\lambda}_{-}\left[\bar{\lambda}_{-}(m-1)+2+\tau-m\right]\left(\bar{\lambda}_{-}^{2} \psi^{2}+\psi^{\prime 2}\right) \psi^{2} \geq 0
\end{aligned}
$$

by (25). Finally, if $\mu=0$, then

$$
j(\psi) \geq\left\{(m-1)\left|\bar{\lambda}_{-}\right|^{m}+(m-2-\tau)\left|\bar{\lambda}_{-}\right|^{m-1}-\bar{a}_{0}\right\}|\psi|^{m} \geq 0
$$

by $(25)$.

Now, note that solutions of $(\overline{S t L})$ are determined uniquely up to a scalar multiple. Therefore we can consider the solution $\psi(\omega)$ normed by the condition

$$
\psi\left(-\frac{\omega_{0}}{2}\right)=1
$$

Lemma 4.2. Let $\psi(\omega)$ be an eigenfunction of the Sturm-Liouville boundary problem $(\overline{S t L})$. Suppose, in addition, that $m \geq 2$ and the inequality

$$
\left|\lambda_{-}\right|^{m}(q+m-1+\mu)+\left|\lambda_{-}\right|^{m-1}(m-\tau-2)>a_{0}
$$

holds. Then $\psi(\omega)>0$ in $\left[-\frac{\omega_{0}}{2}, \frac{\omega_{0}}{2}\right]$ and $\psi^{\prime \prime}(\omega)<0$ in $\left(-\frac{\omega_{0}}{2}, \frac{\omega_{0}}{2}\right)$.

Proof. We rewrite the $(\overline{S t L})$ equation in the form (26). By setting $\bar{y}(\omega)=\frac{\psi^{\prime}(\omega)}{\psi(\omega)}$, we arrive at the problem for $\bar{y}(\omega), \bar{\lambda}_{-}$:

$$
\left\{\begin{array}{l}
\left\{(m-1) \bar{y}^{2}+\bar{\lambda}_{-}^{2}\right\}\left(\bar{\lambda}_{-}^{2}+\bar{y}^{2}\right)^{\frac{m-4}{2}} \bar{y}^{\prime}+(m-1+\bar{\mu})\left(\bar{\lambda}_{-}^{2}+\bar{y}^{2}\right)^{\frac{m}{2}} \\
+\bar{\lambda}_{-}(2+\tau-m)\left(\bar{\lambda}_{-}^{2}+\bar{y}^{2}\right)^{\frac{m-2}{2}}=\bar{a}_{0}, \quad \omega \in\left(-\frac{\omega_{0}}{2}, \frac{\omega_{0}}{2}\right) \\
\left.\alpha(x)\left(\bar{\lambda}_{-}^{2}+\bar{y}^{2}\right)^{\frac{m-2}{2}} \bar{y}(\omega)\right|_{\omega= \pm \frac{\omega_{0}}{2}}=\mp \gamma_{ \pm} .
\end{array}\right.
$$


BEST POSSIBLE ESTIMATES OF WEAK SOLUTIONS OF BOUNDARY VALUE PROBLEMS FOR QUASI-LINEAR ELLIPTIC EQUATIONS IN UNBOUNDED

From the equation of (30) we get:

$$
\begin{gathered}
-\left\{(m-1) \bar{y}^{2}+\bar{\lambda}_{-}^{2}\right\}\left(\bar{\lambda}_{-}^{2}+\bar{y}^{2}\right)^{\frac{m-4}{2}} \bar{y}^{\prime} \\
\geq\left(\bar{\lambda}_{-}^{2}+\bar{y}^{2}\right)^{\frac{m-2}{2}}\left[\bar{\lambda}_{-}^{2}(m-1+\bar{\mu})+\bar{\lambda}_{-}(2+\tau-m)\right]-\bar{a}_{0} \\
\geq\left|\bar{\lambda}_{-}\right|^{m}(m-1+\bar{\mu})+\left|\bar{\lambda}_{-}\right|^{m-1}(m-\tau-2)-\bar{a}_{0}>0
\end{gathered}
$$

by virtue of (29). Thus, it is proved that $\bar{y}^{\prime}(\omega)<0, \omega \in\left(-\frac{\omega_{0}}{2}, \frac{\omega_{0}}{2}\right)$. Therefore $\bar{y}(\omega)$ decreases on the interval $\left(-\frac{\omega_{0}}{2}, \frac{\omega_{0}}{2}\right)$. Therefore

$$
\bar{y}\left(-\frac{\omega_{0}}{2}\right)>\bar{y}(\omega)>\bar{y}\left(\frac{\omega_{0}}{2}\right), \quad \omega \in\left(-\frac{\omega_{0}}{2}, \frac{\omega_{0}}{2}\right)
$$

and hence it follows that $\psi(\omega) \neq 0$ for all $\omega \in\left[-\frac{\omega_{0}}{2}, \frac{\omega_{0}}{2}\right]$. By $(28) \psi(\omega)>0$ in $\left[-\frac{\omega_{0}}{2}, \frac{\omega_{0}}{2}\right]$. Finally, from Lemma $4.1, \psi^{\prime \prime}(\omega)<0$ in $\left[-\frac{\omega_{0}}{2}, \frac{\omega_{0}}{2}\right]$.

Now our aim is to estimate $\psi(\omega)$.

Lemma 4.3. Let $\psi(\omega)$ be an eigenfunction of the Sturm-Liouville boundary problem $(\overline{S t L)}$ and $m \geq 2$. Then

$$
\psi(\omega) \geq \exp \left(-\omega_{0} \gamma_{+}^{\frac{1}{m-1}}\right) .
$$

Proof. First of all, by the definition of $\bar{y}(\omega)$, we have

$$
\psi(\omega)=\exp \left(\int_{-\frac{\omega_{0}}{2}}^{\omega} \bar{y}(\xi) d \xi\right), \quad \omega \in\left(-\frac{\omega_{0}}{2}, \frac{\omega_{0}}{2}\right) .
$$

By the boundary condition of (30), we have

$$
\begin{aligned}
\gamma_{ \pm}=\left|\bar{y}\left( \pm \frac{\omega_{0}}{2}\right)\right| \cdot\left[\bar{\lambda}_{-}^{2}+\bar{y}^{2}\left( \pm \frac{\omega_{0}}{2}\right)\right]^{\frac{m-2}{2}} \geq\left|\bar{y}\left( \pm \frac{\omega_{0}}{2}\right)\right|^{m-1} & \\
& \Rightarrow\left|\bar{y}\left( \pm \frac{\omega_{0}}{2}\right)\right| \leq \gamma_{ \pm}^{\frac{1}{m-1}} .
\end{aligned}
$$

Hence, again by the boundary conditions, it follows that:

$$
-\gamma_{+}^{\frac{1}{m-1}} \leq-\left|\bar{y}\left(\frac{\omega_{0}}{2}\right)\right|=\bar{y}\left(\frac{\omega_{0}}{2}\right)<0 \quad \text { and } \quad 0<\bar{y}\left(-\frac{\omega_{0}}{2}\right) \leq \gamma_{-}^{\frac{1}{m-1}} .
$$

Moreover, in virtue of the decrease of $\bar{y}(\omega)$,

$$
-\gamma_{+}^{\frac{1}{m-1}} \leq \bar{y}(\omega) \leq \gamma_{-}^{\frac{1}{m-1}}, \quad \omega \in\left[-\frac{\omega_{0}}{2}, \frac{\omega_{0}}{2}\right] .
$$

From (33) - (35) the required (32) follows. 
BEST POSSIBLE ESTIMATES OF WEAK SOLUTIONS OF BOUNDARY VALUE PROBLEMS FOR QUASI-LINEAR ELLIPTIC EQUATIONS IN UNBOUNDED

By integrating (30) with regard to $\overline{\lambda_{-}}=\frac{\lambda_{-}}{\zeta}, \bar{a}_{0}=a_{0} \zeta^{1-m}, \bar{\mu}=\mu \zeta$ and performing the change of the variable $\bar{y}(\omega)=\frac{y(\omega)}{\zeta}$, we find that the eigenvalue $\lambda$ satisfies the system

$$
\left\{\begin{array}{l}
y\left(-\frac{\omega_{0}}{2}\right) \frac{\left\{(m-1) y^{2}+\lambda_{-}^{2}\right\}\left(\lambda_{-}^{2}+y^{2}\right)^{\frac{m-4}{2}} d y}{y\left(\frac{\omega_{0}}{2}\right)} \frac{(m-1+q+\mu)\left(\lambda_{-}^{2}+y^{2}\right)^{\frac{m}{2}}+\lambda_{-}(2+\tau-m)\left(\lambda_{-}^{2}+y^{2}\right)^{\frac{m-2}{2}}-a_{0}}{(m)}=\omega_{0} \\
\left.\left(\lambda_{-}^{2}+y^{2}\right)^{\frac{m-2}{2}} y(\omega)\right|_{\omega= \pm \frac{\omega_{0}}{2}}=\mp \gamma_{ \pm},
\end{array}\right.
$$

where $a_{0} \geq 0, \mu>0, q \geq 0, m>1, \tau \leq m-2, \gamma_{ \pm}>0$. We may integrate system $(\Lambda)$ for $m=2$ or $a_{0}=0$. In fact, by integrating of this system we obtain the following.

$$
m=2
$$

In this case we have the system:

$$
\left\{\begin{array}{l}
\int_{y\left(\frac{\omega_{0}}{2}\right)}^{y\left(-\frac{\omega_{0}}{2}\right)} \frac{d y}{(1+q+\mu)\left(\lambda_{-}^{2}+y^{2}\right)+\tau \lambda_{-}-a_{0}}=\omega_{0} \\
\left.y(\omega)\right|_{\omega= \pm \frac{\omega_{0}}{2}}=\mp \gamma_{ \pm} .
\end{array}\right.
$$

Let us define the value $\Upsilon_{-}=\sqrt{\lambda_{-}^{2}+\frac{\tau \lambda_{-}-a_{0}}{1+q+\mu}}$. Then $\lambda_{-}$is the largest negative root of the system

$$
\left\{\begin{array}{c}
\frac{\gamma_{-}+\gamma_{+}}{\zeta \Upsilon_{-}}=\tan \left\{\omega_{0}(1+q+\mu) \Upsilon_{-}\right\} \\
\lambda_{-}<\frac{-\tau-\sqrt{\tau^{2}+4 a_{0}(1+q+\mu)}}{2(1+q+\mu)} \\
a_{O}=0
\end{array}\right.
$$

Let us define the value

$$
\Lambda_{-}=\sqrt{\lambda_{-}^{2}+\frac{\lambda_{-}(2+\tau-m)}{m-1+q+\mu}} .
$$


BEST POSSIBLE ESTIMATES OF WEAK SOLUTIONS OF BOUNDARY VALUE PROBLEMS FOR QUASI-LINEAR ELLIPTIC EQUATIONS IN UNBOUNDED

Then our system $(\Lambda)$ takes the form

$$
\left\{\begin{array}{l}
\frac{2-m}{2-m+\tau}\left\{\arctan \frac{y\left(-\frac{\omega_{0}}{2}\right)}{\lambda_{-}}-\arctan \frac{y\left(\frac{\omega_{0}}{2}\right)}{\lambda_{-}}\right\} \\
+\frac{\frac{m-1}{m-1+q+\mu}-\frac{\lambda_{-}(2-m)}{2-m+\tau}}{\Lambda_{-}}\left\{\arctan \frac{y\left(-\frac{\omega_{0}}{2}\right)}{\Lambda_{-}}-\arctan \frac{y\left(\frac{\omega_{0}}{2}\right)}{\Lambda_{-}}\right\}=\omega_{0} \\
\left.\left(\lambda_{-}^{2}+y^{2}\right)^{\frac{m-2}{2}} y(\omega)\right|_{\omega= \pm \frac{\omega_{0}}{2}}=\mp \gamma_{ \pm} .
\end{array}\right.
$$

Lemma 4.4. Let $\psi(\omega)$ be an eigenfunction of the Sturm-Liouville boundary problem $(\overline{S t L})$ and $m \geq 2$. Then

$$
\psi(\omega) \leq \exp \left\{\frac{q+m-1}{m-1} \cdot \omega_{0} \Lambda_{-} \tan \left[(m-1+q+\mu) \omega_{0} \Lambda_{-}\right]\right\} .
$$

Proof. Because $m \geq 2, a_{0} \geq 0$ and (36), from system $(\Lambda)$ it follows that

$$
\begin{aligned}
\omega_{0} \geq \int_{y\left(\frac{\omega_{0}}{2}\right)}^{y\left(-\frac{\omega_{0}}{2}\right)} \frac{d y}{(m-1+q+\mu)\left(\lambda_{-}^{2}+y^{2}\right)+\lambda_{-}(2+\tau-m)} \\
=\frac{1}{m-1+q+\mu} \int_{y\left(\frac{\omega_{0}}{2}\right)}^{y\left(-\frac{\omega_{0}}{2}\right)} \frac{d y}{y^{2}+\Lambda_{-}^{2}} \\
=\frac{1}{(m-1+q+\mu) \Lambda_{-}}\left\{\arctan \frac{y\left(-\frac{\omega_{0}}{2}\right)}{\Lambda_{-}}-\arctan \frac{y\left(\frac{\omega_{0}}{2}\right)}{\Lambda_{-}}\right\} \\
\geq \frac{1}{(m-1+q+\mu) \Lambda_{-}} \arctan \frac{y\left(-\frac{\omega_{0}}{2}\right)}{\Lambda_{-}} .
\end{aligned}
$$

Hence we get

$$
y\left(-\frac{\omega_{0}}{2}\right) \leq \Lambda_{-} \cdot \tan \left[(m-1+q+\mu) \omega_{0} \Lambda_{-}\right]
$$

Now, from (31), (33) and (38) we derive the required (37).

Corollary 4.5. Let $\psi(\omega)$ be an eigenfunction of the Sturm-Liouville boundary problem $(\overline{S t L})$ and $m \geq 2$. Then

$$
\begin{aligned}
\psi_{0} & \equiv \exp \left(-\omega_{0} \gamma_{+}^{\frac{1}{m-1}}\right) \leq \psi(\omega) \\
& \leq \exp \left\{\frac{q+m-1}{m-1} \cdot \omega_{0} \Lambda_{-} \tan \left[(m-1+q+\mu) \omega_{0} \Lambda_{-}\right]\right\} \equiv \Psi_{0}
\end{aligned}
$$


BEST POSSIBLE ESTIMATES OF WEAK SOLUTIONS OF BOUNDARY VALUE PROBLEMS FOR QUASI-LINEAR ELLIPTIC EQUATIONS IN UNBOUNDED

Lemma 4.6. Let $\psi(\omega)$ be an eigenfunction of the Sturm-Liouville boundary problem $(\overline{S t L})$ and $m \geq 2$. Then for $\omega \in\left[-\frac{\omega_{0}}{2}, \frac{\omega_{0}}{2}\right]$ :

$$
-\zeta^{-1} \gamma_{+}^{\frac{1}{m-1}} \Psi_{0} \leq \psi^{\prime}(\omega) \leq \zeta^{-1} \Lambda_{-} \cdot \tan \left[(m-1+q+\mu) \omega_{0} \Lambda_{-}\right] \Psi_{0} .
$$

Proof. In fact, because $\psi^{\prime}(\omega)=\bar{y}(\omega) \psi(\omega), \psi(\omega)>0, \bar{y}(\omega)=\zeta^{-1} y(\omega)$ and (31) we have

$$
\bar{y}\left(\frac{\omega_{0}}{2}\right) \psi(\omega) \leq \psi^{\prime}(\omega) \leq \zeta^{-1} y\left(-\frac{\omega_{0}}{2}\right) \psi(\omega), \zeta=\frac{m-1}{q+m-1}, \omega \in\left[-\frac{\omega_{0}}{2}, \frac{\omega_{0}}{2}\right] .
$$

Next we use inequalities (34), (38) and Corollary 4.5. Thus we obtain the required statement.

\section{Estimates of the $(B V P)$ solution modulus}

Eventually we can estimate $|u(x)|$ for the problem $(B V P)$ near the infinity.

Theorem 5.1. Let $u$ be a weak solution of the problem $(B V P)$ with $\gamma\left( \pm \frac{\omega_{0}}{2}\right)>$ $\gamma_{ \pm}>0$. Let assumptions (1)-(2) with $m \geq 2$ be satisfied and $\lambda_{-}$be the largest negative number satisfying the system $(\bar{\Lambda})$. Then there exist $d \gg 1$ and $a$ constant $C_{0}$ independent of $u$ such that

$$
|u(x)| \leq C_{0} r^{\lambda_{-}}, \quad \forall x \in \bar{G}_{d} .
$$

Proof. At first, we perform the function change (2) and will consider the function $v(x)$. For the proof we use the above constructed barrier function $z(x)$ (a solution of $(\overline{M V P})$ ) and apply the comparison principle to $v(x)$ and $z(x)$. We consider the operator $Q$, which is defined by $(9)$.

We will show that $Q(A z, \eta) \geq 0=Q(v, \eta)$ for all non-negative $\eta(x) \in C^{0}\left(\overline{G_{d}}\right) \cap$ $\mathfrak{N}_{m, 0, \tau}^{1}\left(G_{d}\right)$ and some $A>0$. Integrating by parts in the first integral, from the $(\overline{M V P})$ equations, we have

$$
\begin{aligned}
Q(A z, \eta) & =\int_{G_{d}}\left(A^{m-1}\left(\mu-\mu_{0}\right) \zeta^{m} r^{\tau} z^{-1}|\nabla z|^{m}-f(x)\right) \eta(x) d x \\
& +\int_{\Gamma_{d}} \alpha(x)\left\{A^{m-1}(\gamma(\omega)-\gamma) r^{\tau-m+1} z|z|^{m-2}-g(x)\right\} \eta(x) d s .
\end{aligned}
$$

Further, by (23) and Corollary 4.5, we obtain

$$
\begin{gathered}
r^{\bar{\lambda}_{-}} \psi_{0} \leq r^{\bar{\lambda}_{-}} \psi(\omega) \leq r^{\bar{\lambda}_{-}} \Psi_{0} \\
|\nabla z|^{2}=z_{r}^{2}+r^{-2} z_{\omega}^{2}=r^{2 \bar{\lambda}_{-}-2}\left(\bar{\lambda}_{-}^{2} \psi^{2}+\psi^{\prime 2}\right) \Longrightarrow\left|\frac{\nabla z}{z}\right| \geq-\bar{\lambda}_{-} r^{-1} .
\end{gathered}
$$


BEST POSSIBLE ESTIMATES OF WEAK SOLUTIONS OF BOUNDARY VALUE PROBLEMS FOR QUASI-LINEAR ELLIPTIC EQUATIONS IN UNBOUNDED

Therefore from (40) according to the above inequalities and because of our assumptions it follows that

$$
\begin{aligned}
Q(A z, \eta) & \geq \int_{G_{d}}\left(A^{m-1}\left(\mu-\mu_{0}\right) \zeta^{m} r^{\tau} z^{-1}|\nabla z|^{m}-f_{1} r^{\tau-m+(m-1) \bar{\lambda}_{-}}\right) \eta(x) d x \\
& +\int_{\Gamma_{d}}\left(A^{m-1}(\gamma(\omega)-\gamma) r^{\tau-m+1} z^{m-1}-g_{1} r^{\tau-m+1+(m-1) \bar{\lambda}_{-}}\right) \eta(x) d s \\
& \geq\left(\left|\bar{\lambda}_{-}\right|^{m}\left(A \psi_{0}\right)^{m-1}\left(\mu-\mu_{0}\right) \zeta^{m}-f_{1}\right) \int_{G_{d}} r^{\tau-m+(m-1) \bar{\lambda}_{-}} \eta(x) d x \\
& +\left(\left(A \psi_{0}\right)^{m-1}(\gamma(\omega)-\gamma)-g_{1}\right) \int_{\Gamma_{d}} r^{\tau-m+1+(m-1) \bar{\lambda}_{-}} \eta(x) d s .
\end{aligned}
$$

Now, taking into consideration that $\mu>\mu_{0}, \gamma\left( \pm \frac{\omega_{0}}{2}\right)>\gamma$ and choosing $A>0$ sufficiently large: $A \geq \frac{1}{\psi_{0}} \max \left\{\left(\frac{f_{1}}{\left|\lambda_{-}\right|^{m}\left(\mu-\mu_{0}\right)}\right)^{\frac{1}{m-1}},\left(\frac{g_{1}}{\gamma\left( \pm \frac{\omega_{0}}{2}\right)-\gamma_{ \pm}}\right)^{\frac{1}{m-1}}\right\}$, we provide the required inequality $Q(A z, \eta) \geq 0=Q(v, \eta)$.

Now, by the continuity of $v(x)$ and by Theorem 3.1, we have $\left.v(x)\right|_{\Omega_{d}} \leq M_{0}^{1 / \zeta}=$ $\max _{\bar{G}}|v(x)|$. On the other hand, by virtue of (41), $\left.A z\right|_{\Omega_{d}} \geq A d^{\bar{\lambda}_{-}} \psi_{0} \geq M_{0}^{1 / \zeta} \geq$ $\left.v\right|_{\Omega_{d}} ^{G}$ provided that $A \geq\left(d^{\bar{\lambda}_{-}} \psi_{0}\right)^{-1} \cdot M_{0}^{1 / \zeta}$. Thus from above we get

$$
\left\{\begin{array}{l}
Q(A z, \eta) \geq Q(v, \eta), \quad \forall \eta \in C^{0}\left(\overline{G_{d}}\right) \cap \mathfrak{N}_{m, 0, \tau}^{1}\left(G_{d}\right) \\
\left.A z\right|_{\Omega_{d}} \geq\left. v\right|_{\Omega_{d}}
\end{array}\right.
$$

All conditions of the comparison principle are fulfiled, so by this we get

$$
v(x) \leq A z(r, \omega), \quad \forall x \in \bar{G}_{d} .
$$

Similarly, $v(x) \geq-A z(r, \omega)$ for all $x \in \bar{G}_{d}$. Thus, finally, we obtain

$$
|v(x)| \leq A z(r, \omega) \leq c_{0} r^{\bar{\lambda}_{-}}, \quad \forall x \in \bar{G}_{d} ; \quad c_{0}=A \Psi_{0},
$$

by (41). On returning to the old variables, by virtue of (2), we get the desired estimate (39).

\section{References}

[1] Borsuk, M.V.: Transmission problems for elliptic second-order equations in non-smooth domains. Birkhäuser Basel book (2010) 
BEST POSSIBLE ESTIMATES OF WEAK SOLUTIONS OF BOUNDARY VALUE PROBLEMS FOR QUASI-LINEAR ELLIPTIC EQUATIONS IN UNBOUNDED

[2] Borsuk, M.V., Wiśniewski, D.: Boundary value problems for quasilinear elliptic second order equations in unbounded cone-like domains, Cent.Eur.J.Math., (2012), 2051-2072

[3] Cirmi, G.R., Porzio, M.M.: $L^{\infty}$ - solutions for some nonlinear degenerate elliptic and parabolic equations, Ann. mat. pura ed appl. (IV) 169 (1995), $67-86$

[4] Drabek, P., Kufner, A., Nicolosi, F.: Quasilinear elliptic equations with degenerations and singularities. Walter de Gruyter. Berlin, NY, 1997

[5] Furusho, Ya.: Existence of global positive solutions of quasilinear elliptic equations in unbounded domains, Funkcialaj Ekvacioj, 32 (1989), 227-242

[6] Hernández, J., Mancebo, F.J., Vega, J.M.: On the linearization of some singular, nonlinear elliptic problems and applications, Ann. Inst. H. Poincaré Anal.Non Linéaire 19 (2002), 777-813.

[7] Kondratiev, V., Liskevich, V., Moroz, V.: Positive solutions to superlinear second-order divergence type elliptic equations in cone-like domains, Ann. Inst. H. Poincare Anal. Non Lineaire, 22 (2005), 25-43.

[8] Lazer, A.C., McKenna, P.J.: On a singular nonlinear elliptic boundaryvalue problem, Proc. Amer. Math. Soc. 111 (1991), 721-730.

[9] Mityushev, V., Adler, P.: Darcy flow around a two-dimensional lens, I. Phys. A: Math. Gen. 39, 2006, 3545-3560.

[10] Murray, J.D.: Mathematical Biology, Springer, Berlin (1993).

[11] Murthy, M.K.V., Stampacchia, G.: Boundary value problem for some degenerate elliptic operators, Ann. Math. Pura Appl. 80 (Ser. IV) (1968)

[12] Nachman, A., Callegari, A.: A nonlinear singular boundary value problem in the theory of pseudoplastic fluids, SIAM J. Appl. Math. 38(1980), 275281.

[13] Noussair, E.S., Swanson, C.A.: Decaying entire solutions of quasilinear elliptic equations, Funkcialaj Ekvacioj, 31 (1988), 415-438

[14] Okubo, A., Levin, S.A.: Diffusion and Ecological Problems: Modern Prospectives, Springer, New York (2001).

[15] Ouassarah, A.A., Hajjaj, A.: Existence of solutions for quasilinear elliptic boundary value problems in unbounded domains, Bull. Belg. Math. Soc. 3 (1996), 217-225. 
BEST POSSIBLE ESTIMATES OF WEAK SOLUTIONS OF BOUNDARY VALUE PROBLEMS FOR QUASI-LINEAR ELLIPTIC EQUATIONS IN UNBOUNDED

[16] Pao, C.V.: Nonlinear elliptic boundary-value problems in unbounded domains, Nonlinear Analysis, Theory, Methods and Applications, Vol. 18, No. 8 (1992), 759-774

[17] Shilov, G.E., Gel'fand, I.M.: Generalized functions, 1964, v. I. Academic Press

[18] Wiśniewski, D.: Boundary value problems for a second-order elliptic equation in unbounded domains, Ann. Univ. Paed. Cracov. Studia Math. IX (2010)

Damian WiŚNIEWSKI,

Faculty of Mathematics and Computer Science,

University of Warmia and Mazury in Olsztyn,

Sloneczna 54, 10-710 Olsztyn, Poland.

Email: dawi@matman.uwm.edu.pl 